\title{
Self diffusion studies on cobalt thin films
}

\author{
J J B PRASAD and $K$ V REDDY \\ Department of Physics, Indian Institute of Technology. Madras 600036, India \\ MS received 6 November 1984
}

\begin{abstract}
The study of lateral diffusion in thin metallic films is important from the application point of view, especially in electromigration reliability studies. Lateral self diffusion in cobalt thin films is studied using a non-destructive tracer scanning method. Neutron irradiation is employed to make a well-defined radioactive $\left({ }^{60} \mathrm{Co}\right)$ region in the middle of a continuous cobalt thin film stripe of width $3 \mathrm{~mm}$. The experimental data are fitted to the appropriate solution of the diffusion equations by means of a non-linear least square fitting procedure using a computer. The diffusion experiments are conducted in the temperature range $300-600^{\circ} \mathrm{C}$ in argon atmosphere. This thin film data are compared with the diffusion data available on bulk cobalt. The activation energy for surface diffusion obtained $(0.14 \mathrm{eV})$ is very much smaller than the reported activation energy for grain boundary diffusion in cobalt.
\end{abstract}

Keywords. Cobalt; thin films; surface diffusion.

\section{Introduction}

With the increasing utility of thin films for various applications such as solar cells, strain gauges, semiconductor devices etc., their studies have acquired momentum in recent years. Diffusion studies in thin films are important to the device engineers due to its dominant role played in determining the reliability of the metallization in planar semiconductor devices. In some of the failure modes such as electromigration, thermomigration etc., the parameters like effective charge are controlled by the diffusion processes. From the fundamental point of view also, these studies are interesting because the mechanism of diffusion here is different from that of the bulk materials and depends on the way in which the sample is prepared.

The diffusion data available on thin films are meagre compared to the data on bulk polycrystalline and single crystal materials. One of the reasons for this is probably the lack of proper experimental technique for these studies. The methods that are adopted in bulk samples such as microtoming, grinding etc., cannot be used in the case of thin films. Impurity diffusion in thin film couples has been studied in recent years using sputter ion mass spectrometry (McHugh 1975), Auger electron spectroscopy (Chang and Quintana 1976), electron probe micro analysis (Ho and Howard 1975) etc. Most of these studies are normal diffusion studies. Self diffusion in gold thin films in the normal direction has been studied by Gupta and Asai (1974) using tracers and $r-f$ sputtering method. Beniere et al (1978) have used a sectioning technique to determine lateral diffusion of copper in aluminium thin films using tracers. The present authors have developed a non-destructive tracer scanning technique to study diffusion and electromigration in thin films and this has been used in indium thin films also (Prasad and Reddy 1983a). In this paper the results on lateral self diffusion studies in cobalt thin films using the neutron activation-tracer scanning (NATS) method are reported. 
Vacuum deposited polycrystalline thin films consist of regions with high density of defects such as grain boundaries, surfaces etc. in addition to the point defects inside the grain. These extended defects provide fast and efficient short circuiting paths for mass transport. They can be classified as grain boundary diffusion and surface diffusion. Diffusion along grain boundaries in polycrystalline thin films is several orders of magnitude more rapid than the lattice diffusion due to point defects. In most of the earlier studies on diffusion in thin films (Sun and Ohring 1976; Reddy et al 1979; Prasad and Reddy 1983a) this mechanism is observed on comparing the bulk and thin film data. Diffusion due to surfaces is also possible in thin metallic films. Here diffusion of atoms can take place along external and internal surfaces. The rate of diffusion is faster in surface diffusion since the activation energy required is very low. This type of mass transport is observed (Van Gurp 1976) by indirect means in the case of cobalt films by observing grain boundary grooving and thermal faceting. No systematic study has been made to confirm the surface mechanism of mass transport in any material in the thin film form.

\section{Experimental}

Lateral self diffusion in cobalt thin films is studied using NATs method. The shape of the experimental samples used in this study is shown in figure la. Samples are prepared in two stages. In the first stage 99.999 pure cobalt is evaporated using a $3 \mathrm{~kW}$ electron gun in a vacuum coating unit at $(1-5) \times 10^{-6}$ torr over an area of $3 \times 1 \mathrm{~mm}$, the thickness being $0.0001 \mathrm{~mm}$. Oxidised silicon single crystal wafers $(250 \mu$ thick) are used as substrates which are heated to $250^{\circ} \mathrm{C}$ during evaporation to get good adhesion and better films.

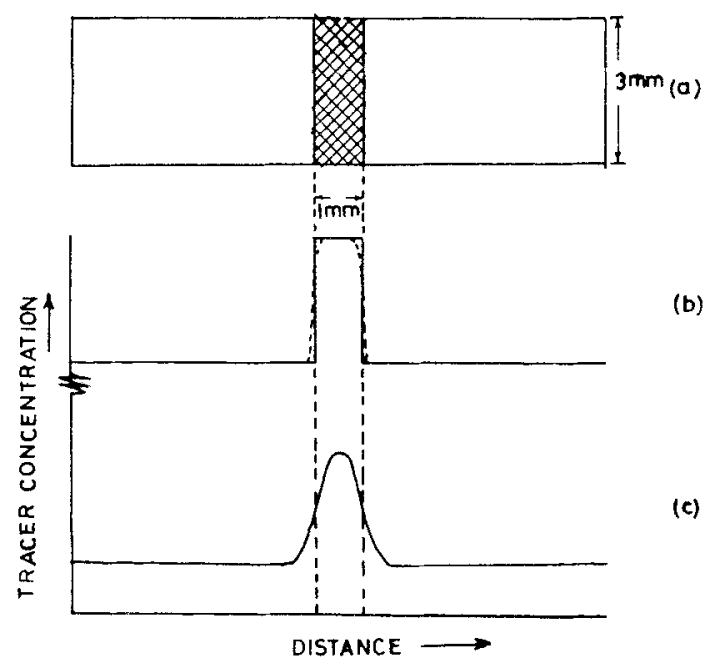

Figure 1. . Schematic diagram of the experimental sample used for diffusion studies. Shaded region contains radioactive cobalt; continuous stripe pattern is ordinary cobalt film. b. Radioactive cobalt concentration profile of the sample before diffusion annealing; continuous line represents the ideal case when the profile is taken with an infinitely small slit width; dotted line represents practical profile when viewed through a slit of finite width. c. Shape of the tracer profile after diffusion annealing. 
Samples containing the cobalt films were irradiated in CIRUS reactor at BARC, Bombay with thermal neutrons. Neutron flux employed is $10^{13}$ neutrons $/ \mathrm{cm}^{2} \mathrm{sec}$ and the period of irradiation was one week. On neutron irradiation ${ }^{59} \mathrm{Co}$ captures a neutron to form radioactive ${ }^{60} \mathrm{Co}$ isotope with a half-life of 5.27 years. The fraction of ${ }^{60} \mathrm{Co}$ radioactive isotope formed after thermal neutron irradiation in the present study is estimated to be $6 \mathrm{ppm}$. The background activity due to silicon substrates decays to negligible level after storing the samples for 2-3 days after irradiation.

Continuous cobalt thin film strips $(10 \times 3 \times 0.0004 \mathrm{~mm})$ is then evaporated on to the radioactive cobalt regions (figure 1a). Metal contact masks made by photolithography are used for obtaining samples of well-defined edges.

Diffusion experiments were conducted in argon atmosphere. Samples were introduced into a quartz tube which was evacuated and filled with argon at $1 / 3 \mathrm{~atm}$. pressure. This tube was introduced into a furnace, the temperature of which was controlled within $\pm 0.5^{\circ} \mathrm{C}$. Diffusion experiments were conducted in the temperature range $300-600^{\circ} \mathrm{C}$.

Radioactive cobalt concentration profiles in the experimental samples were obtained by a tracer scanning method. The details of this technique are mentioned in detail elsewhere (Prasad and Reddy 1983a). Here the samples were moved horizontally by means of a precision micrometer arrangement in steps of $50 \mu$ and the radioactivity at each position was recorded through an adjustable slit. The width of the slit employed in this case was $50 \mu$ and $\beta$ rays were detected by means of a plastic scintillator and a single channel analyser assembly. Tracer concentration at each position was plotted as a function of distance and the shape of the profile before and after diffusion is shown in figures $1 \mathrm{~b}$ and $1 \mathrm{c}$ respectively.

\section{Results}

In figure $1 \mathrm{~b}$, the solid line represents the shape of the profile in the ideal case when viewed through a slit of infinitely small width. The dotted profile represents the practical profile when scanned through a slit of finite width. The initial slope is due to the finite width of the slit. For the ideal profile, the concentration after diffusion is given by

$$
C(x, t)=\frac{C_{0}}{2}\left[\operatorname{erf}\left(\frac{h+x}{2 \sqrt{D t}}\right)+\operatorname{erf}\left(\frac{h-x}{2 \sqrt{D t}}\right)\right]
$$

where $C(x, t)$ is the concentration at a distance $x$ from the centre of a region of initial width $2 h$ after annealing for time $t$ and $C_{0}$ is the concentration at $x=0 ; D$ is diffusion coefficient. Corresponding to the practical profile (shown in dotted lines) the experimentally observed tracer concentration $C^{\prime}(x, t)$ after diffusion can be obtained from the above equation as

$$
C^{\prime}(x, t)=\int_{x-w / 2}^{x+w / 2} C(x, t) \mathrm{d} x
$$

where $w$ is the slit width. On substituting $C(x, t)$ from (1)

$$
C^{\prime}(x, t)=\frac{C_{0}}{2} \int_{x-w / 2}^{x+w / 2}\left[\operatorname{erf}\left(\frac{h+x}{2 \sqrt{D t}}\right)+\operatorname{erf}\left(\frac{h-x}{2 \sqrt{D t}}\right)\right] \mathrm{d} x .
$$


The value of the diffusion coefficient $D$ is obtained by fitting the experimental data to the above equation numerically. Non-linear least squares method is employed for fitting using a computer. The corrections due to statistical errors in the radioactive counting while recording the data are also incorporated in the programme. The best fitted values are taken as the $D$ values at the respective temperatures.

Diffusion experiments are conducted on cobalt thin films in the temperature range $300-600^{\circ} \mathrm{C}$. Figure 2 shows the lateral self diffusion profile of a cobalt thin film sample annealed for $72 \mathrm{hr}$ at $400^{\circ} \mathrm{C}$. The $D$ value at this temperature is $4.48 \times 10^{-10} \mathrm{~cm}^{2} / \mathrm{sec}$. The results obtained are given in table 1 . The experimental data on lateral self diffusion coefficients in cobalt thin films are found to obey the Arrhenius law. The data could be

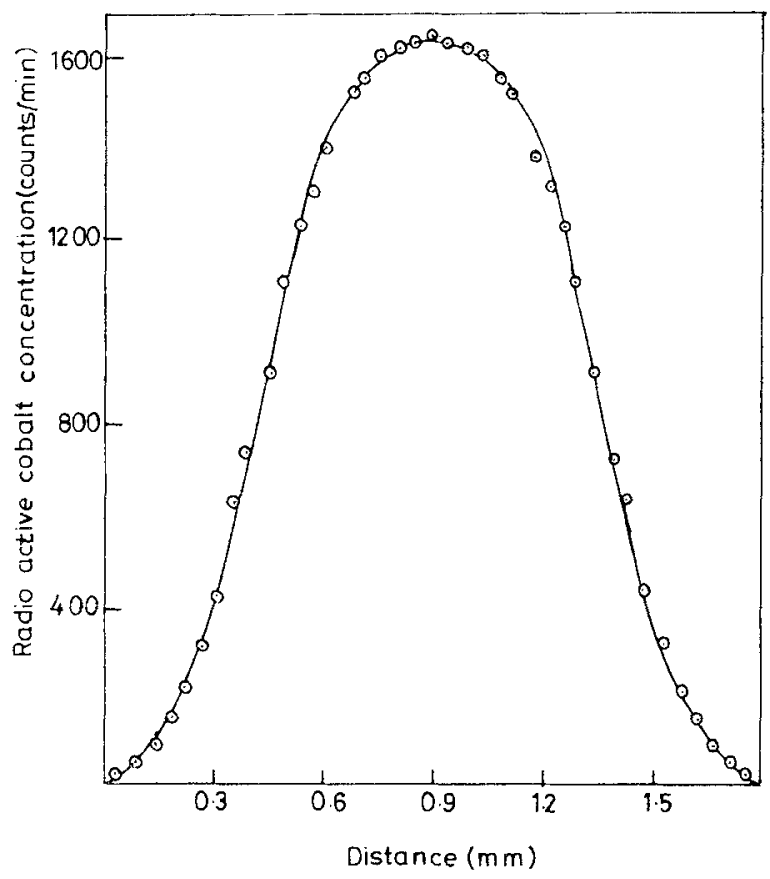

Figure 2. Lateral self diffusion profile of a cobalt thin film sample annealed at $400^{\circ} \mathrm{C}$ for $72 \mathrm{hr}$. Circles represent experimental points and continuous line represents fitted profile.

Table 1. Experimental results on lateral self diffusion in cobalt thin films.

\begin{tabular}{ccc}
\hline $\begin{array}{c}\text { Temperature } \\
\left({ }^{\circ} \mathrm{C}\right)\end{array}$ & $\begin{array}{c}\text { Time of anneal } \\
(\mathrm{hr})\end{array}$ & $\begin{array}{c}\text { Diffusion } \\
\text { coefficient } \times 10^{-10} \\
\left(\mathrm{~cm}^{2} / \mathrm{sec}\right)\end{array}$ \\
\hline 300 & 126 & 3.74 \\
400 & 72 & 4.48 \\
500 & 110 & 5.39 \\
550 & 110 & 6.85 \\
600 & 70 & 8.74 \\
\hline
\end{tabular}




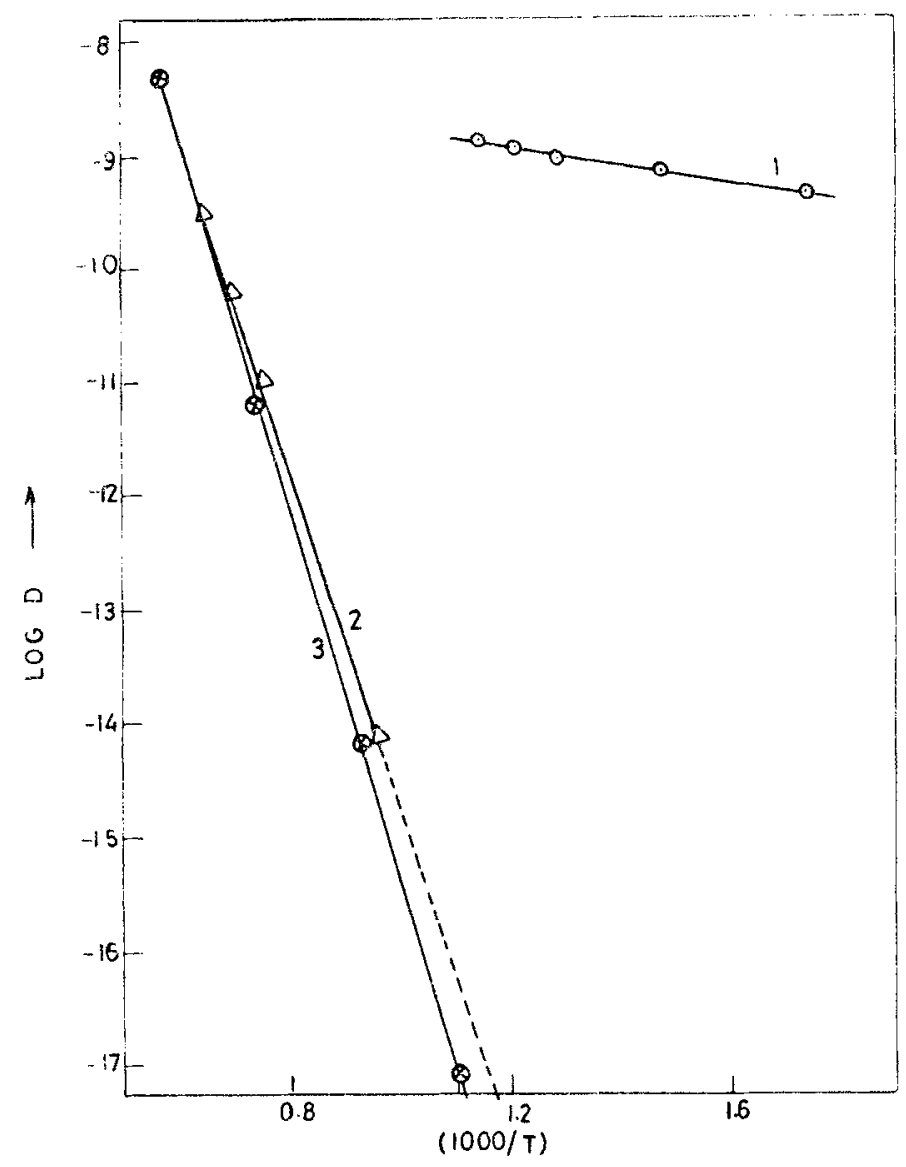

Figure 3. Arrhenius plot of the diffusion data in cobalt. 1. Present data on thin films 2. Hirano et al (1962) data on bulk cobalt 3. Bussmann et al (1979) data on high purity bulk cobalt.

fitted to a single straight line satisfying the equation

$$
D=[0.48 \pm 0.04] \times 10^{-8} \exp [(0.14 \pm 0.008) \mathrm{eV} / \mathrm{kT}] \mathrm{cm}^{2} / \mathrm{sec}
$$

The Arrhenius plot along with available bulk data is given in figure 3 . Error calculations in pre-exponential factor and activation energy values include the errors in the diffusion profile measurement and the accuracy of the fitting procedure.

\section{Discussion}

Self diffusion experiments were done extensively on bulk cobalt by various researchers using tracer techniques (Ruder and Birchenall 1951; Nix and Jan Mott 1951; Mead and Birchenall 1955; Lange et al 1962; Hirano et al 1962; Bussmann et al 1979). All these studies were made at higher temperatures mostly near the melting point of cobalt. Microtoming and $r-f$ sputtering methods were employed for sectioning. Hirano et al (1962) reported pre-exponential factor $\left(D_{0}\right)$ and activation energy $(E)$ values as 
$0.5 \mathrm{~cm}^{2} / \mathrm{sec}$ and $2.84 \mathrm{eV}$ respectively in the temperature range $772-1048^{\circ} \mathrm{C}$. Bussmann et al (1979) made self diffusion studies on high purity cobalt in the temperature range $623-1472^{\circ} \mathrm{C}$. Here again radioactive tracers were employed. The values of $D_{0}$ and $E$ are $0.55 \mathrm{~cm}^{2} / \mathrm{sec}$ and $2.99 \mathrm{eV}$ respectively. Vacancy mechanism of diffusion was concluded from the experimental data. Gleiter and Chalmers (1972) in their review on grain boundary diffusion in solids reported the activation energy and preexponential factor for grain boundary diffusion in bulk cobalt as $1.68 \mathrm{eV}$ and $4 \mathrm{~cm}^{2} / \mathrm{sec}$ respectively.

No other experimental data are available on cobalt thin films to compare our data on lateral self diffusion. In figure 3, our data are compared with the bulk data of Hirano et al (1962) and Bussmann et al (1979). It is clear that our values of $D_{0}$ and $E$ are very much smaller compared to the bulk data. The values are smaller than the grain boundary diffusion data, indicating a possible surface diffusion in cobalt thin films.

Van Gurp (1976) studied the electromigration in cobalt thin films using indirect methods such as resistance monitoring and electron microscope observations. Grain boundary grooving and thermal faceting of the surfaces are observed in this study in the temperature range $400-615^{\circ} \mathrm{C}$. The main reason for this is attributed to the possible surface diffusion in cobalt thin films. These observations lend further support to our contention-namely surface diffusion occurs in cobalt thin films.

The present authors (Prasad and Reddy 1983b) had also studied electromigration in cobalt thin films by a direct method using radioactive tracer ${ }^{60} \mathrm{Co}$ and concluded that the effective charge $Z^{*}$ is independent of temperature. This was attributed to surface diffusion. From the above it could be concluded that surface diffusion is the dominant mechanism.

\section{References}

Beniere F, Reddy K V, Kostopoulos D and Le Traon J Y 1978 J. Appl. Phys. 492743

Bussmann W, Herzig C H, Rempp W, Maier K and Mehrer H 1979 Phys. Status. Solidi a56 87

Chang C C and Quintana G 1976 Appl. Phys. Lett. 29453

Gleiter H and Chalmers B 1972 Prog. Mater. Sci. 577

Gupta D and Asai K W 1974 Thin Solid Films 22121

Hirano K I, Agarwala R P, Averbach B L and Cohen M 1962 J. Appl. Phys. 333049

Ho P S and Howard J K 1975 Appl. Phys. Lett. 27261

Lange W, Haessner A and Siebov K 1962 Isotopentech 242

McHugh J A 1975 Methods of surface analysis (Netherlands: Elsevier) 273

Mead H W and Birchenall C E 1955 J. Metall. 7994

Nix F C and Jan Mott Jr F E 1951 Phys. Rev. 8272

Prasad J J B and Reddy K V 1983a Indian J. Pure Appl. Phys. 21391

Prasad J J B and Reddy K V 1983b 2nd International Workshop on the physics of semiconductor devices,

New Delhi, Abstracts p. 149

Reddy K V, Beniere F, Kostopoulos D and Le Traon J Y 1979 J. Appl. Phys. 502782

Ruder R C and Birchenall C E 1951 J. Metall. 3142

Sun P H and Ohring M 1976 J. Appl. Phys. 47478

Van Gurp G J 1976 Thin Solid Films 38295 